\title{
Does genetic anticipation occur in familial rheumatoid arthritis?
}

\author{
Chris Deighton, Peta Heslop, Janet McDonagh, David Walker, Glenys Thomson
}

\begin{abstract}
Objective-To determine if there is evidence for genetic anticipation in rheumatoid arthritis (RA) by analysing the possibility that parental disease status and age at proband conception influence the age of onset and disease severity of the proband.
\end{abstract}

Method-RA outpatients were identified and data were also taken from Newcastle multicase RA pedigrees. Comparisons of age of onset and parental age at proband conception were made for pedigrees grouped according to the disease status of the parents. Correlation coefficients and linear regression models were calculated for the age of RA onset in the probands. Measures of disease severity were compared in RA mother-proband pairs.

Results-The results were similar in both the outpatient $(n=153)$ and multicase pedigree $(n=15)$ samples. Significant results were confined to pedigrees in which the mother had RA (20 of the outpatient probands and seven of the multicase group). Probands in these sibships had a younger age of RA onset than their affected mothers (38.3 years $(95 \%$ confidence interval (CI) 33.8 to 42.8 ) versus $53.7(47.3$ to 60.0$)(p=0.002)$ in the outpatient sample; 32.4 years $(25.3$ to $39 \cdot 6)$ versus 43.4 years $(29.0$ to 57.9$)(p=0.1)$ in the multicase pedigrees). In the maternal RA group, both the maternal and paternal age at proband conception showed significant negative correlations $(r=$ $-0.65, p=0.002$ and $r=-0.60, p=0.005$, respectively in the outpatient sample) and linear regression coefficients with age of proband disease onset. In seven affected mother-proband pairs, the probands had a tendency to more severe disease, despite shorter disease duration and younger age. Conclusions-This preliminary analysis has suggested that within pedigrees in which the mother has RA, the features of genetic anticipation and observations consistent with premutation models may prevail.

(Ann Rheum Dis 1994; 53: 833-835)

Genetic anticipation describes the tendency in some diseases for successive generations to experience more severe and earlier onset disease. A well documented example is myotonic dystrophy (MD). ${ }^{1} \mathrm{~A}$ molecular correlate for this has recently been described for MD and other diseases such as Huntington's disease and fragile $\mathrm{X}$ syndrome: ${ }^{2}$ tandemly repeated trinucleotide sequences close to or within the disease-associated gene expand, changing from marginally expanded premutant alleles associated with a normal or subclinical phenotype, to large increases in copy numbers, and the fully expressed disease. These diseases all demonstrate genetic anticipation to some degree, with earlier age of disease onset and increasing disease severity over the generations correlating with a progressively expanding trinucleotide repeat sequence. ${ }^{2}$ In both $\mathrm{MD}$ and Huntington's disease it has been demonstrated that paternal age at which the patient was conceived is negatively associated with age of onset of the disease. ${ }^{3}$ While a number of mechanisms may be responsible for this, Zheng et al argue that this reflects the germ cells continuing to divide mitotically (and hence be subject to continued expansion) in the postembryonic state only in males. ${ }^{3}$

Recently, genetic anticipation and premutation phenomena have been described in genetically complex diseases such as bipolar affective disorder, ${ }^{4}$ and psoriasis. ${ }^{5}$ In this preliminary investigation, we have sought evidence for similar observations in RA.

\section{Patients and methods}

Patients with RA fulfilling 1987 American Rheumatism Association criteria ${ }^{6}$ were consecutively identified from outpatient clinics in Nottingham and Newcastle-upon-Tyne, UK. The following information was obtained from each patient: date of birth of the proband and both parents; RA status of the parents as reported by the patient; age of symptomatic disease onset in the proband and the parents where relevant.

For four groups for which neither parent, mother only, father only, or both parents were reported as having RA, we performed the following analyses:

(1) Descriptive statistics were calculated for the probands' age of onset of disease, parental ages at conception of the proband, and parental age of disease onset where relevant. Differences between ages of onset in affected parentoffspring pairs were tested using paired $t$ tests. 
(2) Correlation coefficients were calculated for age of disease onset in the proband compared with paternal and maternal ages at conception of the proband.

(3) Linear regression models were developed to examine if parental ages at proband conception could predict proband age of RA onset independently of parental age of RA onset (where appropriate).

Because the RA status of first degree relatives can be unreliable when ascertained from the patient, ${ }^{7}$ we investigated the same set of hypotheses on parent and proband combinations identified from Newcastle-upon-Tyne RA multicase pedigrees described previously. ${ }^{8}$ Here the diagnosis in each individual had been fully assessed.

The above analyses suggested that older parental ages at conception of the proband correlated negatively with earlier age of onset of RA in the proband only in those pedigrees in which the mother had RA. For each proband affected mother pedigree from the Newcastle multicase pedigrees, the following measures of disease severity were compared within affected pairs: spread/severity index; ${ }^{9}$ Health Assessment Questionnaire (HAQ) score; ${ }^{10}$ IgM rheumatoid factor titre, measured by RAHA (Fugizoki Pharmaceutical, Tokyo, Japan). HLA-DR typing for antigens 1-10 was performed using $\mathrm{B}$ lymphocytes isolated by their adherence to nylon wool columns, and using standard alloantisera. ${ }^{11}$ The influence of differences in age, sex and disease duration were considered.

Table 1 Correlation coefficients for age of proband at disease onset, and parental ages at conception of the proband and disease onset, in groups classified according to the parental disease status

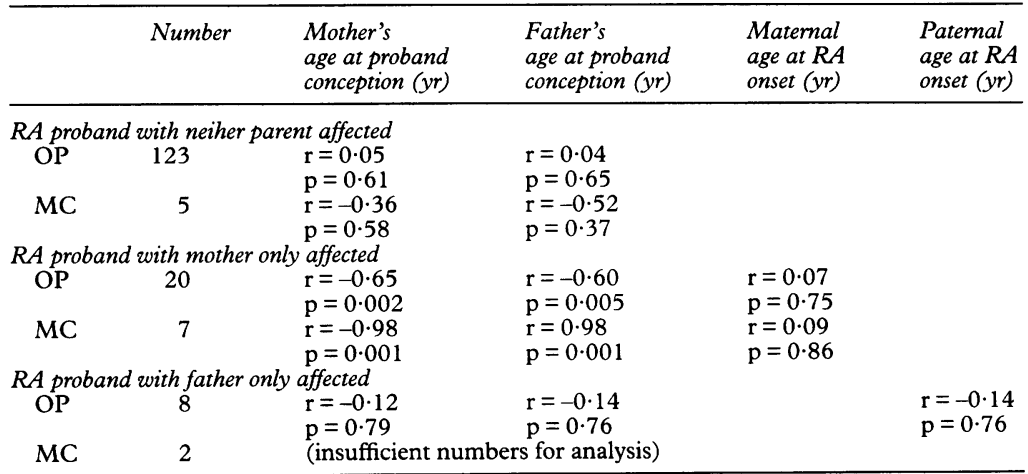

$\mathrm{OP}=$ Outpatient sample; $\mathrm{MC}=$ multicase sample.

Table 2 Linear regression models $\uparrow$ for age of proband at disease onset, and parental ages at conception of the proband and disease onset, in families in which only the mother has rheumatoid arthritis (outpatient and multicase pedigrees combined $(n=27)$ )

\begin{tabular}{lllllr}
\hline Model & Constant & $\begin{array}{l}\text { Mother's } \\
\text { age at RA } \\
\text { onset }(y r)\end{array}$ & $\begin{array}{l}\text { Maternal } \\
\text { age at proband } \\
\text { conception }(y r)\end{array}$ & $\begin{array}{l}\text { Paternal } \\
\text { age at proband } \\
\text { conception }(y r)\end{array}$ & $\begin{array}{l}\text { Model } \\
R^{2} \varsigma\end{array}$ \\
\hline 1 & 57.402 & 0.123 & $\begin{array}{l}-0.939^{\star \star \star} \\
(-1.380 \text { to }-0.498)\end{array}$ & $\begin{array}{l}-0.818^{\star \star \star} \\
(-1.265 \text { to }-0.371)\end{array}$ & 0.456 \\
2 & 55.907 & 0.123 & & & -392 \\
\hline
\end{tabular}

$\dagger$ Linear regression allows predictive models to be developed for the dependent variable of proband age of RA onset, so that in the first model proband age of RA onset $=57 \cdot 402+(0 \cdot 123$ mother's age at RA onset) $-(0.939$ mother's age at proband conception $)$

$R^{2}$ is the amount of variance accounted for by the model. In the first model, $45 \cdot 6 \%$ of the variance of proband age of RA onset can be accounted for by the variables included in the model. Figures in parentheses are the $95 \%$ confidence intervals for those variables with significan predictive value. $\star \star \star \mathrm{p}<0.001$.

\section{Results}

We identified 153 RA patients (131 females, 22 males) from the outpatient clinics in Nottingham and Newcastle. The overall mean age of onset of symptomatic disease was 43.5 years (SD 13.8, range 7-80). The mean paternal age at conception of the proband was $30 \cdot 1$ years (SD $7 \cdot 4$, range $17-57$ ); mean maternal age was $28 \cdot 1$ years $(\mathrm{SD} 6 \cdot 1$, range 16-46).

The ages at which parents conceived the RA proband and (where appropriate) the ages at which disease onset occurred were compared for subpopulations determined by the RA status of the parents. In the outpatient population probands who reported that their mothers had RA $(n=20)$ described an older age of disease onset in their mother $(n=20)$ than they themselves had experienced $(38.3$ years $(95 \%$ CI 33.8 to $42 \cdot 8)$ versus $53 \cdot 7(47 \cdot 3$ to $60 \cdot 0)$ (paired $t$ test, $\mathrm{p}=0 \cdot 002)$ ). All other comparisons in other patient groups were not significant statistically. In the multicase pedigrees, the affected mother-proband pairs $(n=7)$ also showed a trend towards earlier RA onset in the proband (32.4 years versus 43.4 years $(29.0$ to $57 \cdot 9)$ (paired $t$ test, $\mathrm{p}=0 \cdot 1)$ ). Although the probands in the maternally affected pedigrees from the multicase sample had a younger mean age of onset than those in the outpatient sample $(32.4$ years $(25.3$ to 39.6 ) versus 38.3 years $(33.8$ to $42 \cdot 8)$ ), this difference was not significant.

Pearson correlation coefficients were calculated for both data sets (table 1). Significant correlations were confined to the groups in which the mother was affected; inverse correlations were seen for ages of both parents at conception of the proband and the age of proband RA onset.

Table 2 shows linear regression models for probands with affected mothers. None of the models in other parental disease status groups was significant. Similar results were seen in both outpatient and multicase data sets. The full model including both parental ages at proband conception is not shown because the limited data set and the high correlation between these variables $(r=0.08, p<0.001)$ make it difficult to state confidently if maternal, paternal, or both ages at conception of proband are of prime importance for age of onset of disease in the proband.

The affected mothers of probands from the Newcastle multicase pedigrees $(n=7)$ had a longer disease duration (mean duration 29 years versus 15 years), and were obviously older at the time of assessment ( 72 years versus 45 years) than their Nottingham counterparts, but despite this their respective paired probands had a tendency to more severe disease on both the spread/severity index score (17 versus 14$)$ and HAQ score (2.0 versus 1.9$)$. They also tended towards a higher rheumatoid factor titre $(1 / 274$ versus $1 / 180)$. Only one proband was homozygous for HLA-DR4, and two were negative for this antigen. Only four of the seven haplotypes transmitted from affected mother to affected daughter were HLA-DR4 positive. 


\section{Discussion}

In this preliminary investigation, the only subgroup in which we could find any evidence for an effect of parental age at conception of the proband on age of onset of disease in the proband comprised those probands who reported having, or were documented to have, a mother with RA. We collected data on consecutive outpatients whom we questioned about the RA status of their parents. This is known to be unreliable, ${ }^{7}$ therefore we compared the outpatient population with a smaller but fully documented population of multicase pedigrees. Our analyses produced similar results for both data sets.

In the maternal RA subgroup, the following observations are of interest with respect to genetic anticipation and observations in diseases with an unstable trinucleotide repeat:

(1) Probands developed RA at an age that was earlier than the age of disease onset in their affected mother.

(2) The ages of both the mother and the father at conception of the proband correlated negatively with the age of RA onset in the proband, and had predictive value for this variable by linear regression.

(3) In the small number of affected motherproband pairs for whom we had detailed information, the probands appeared to have more severe disease, particularly when their younger age and shorter disease duration were taken into consideration.

The epidemiological phenomenon of genetic anticipation is subject to bias, however, particularly in a retrospective study such as this. There is a bias towards identifying those pedigrees in which RA develops late in the life of the parent and early in the life of the offspring. Pedigrees in which the parent develops early onset RA and the offspring shows late onset disease are less likely to be identified. Further and larger studies are required to address this. The number of families with affected fathers was small and it is therefore difficult to conclude that such features may be unique to maternally affected families. Large numbers of those families with unaffected parents may be needed to detect any influence of parental ages on age of onset, as is the case for Huntington's disease. $^{3}$

Whilst it is easier to visualise the mechanism of premutation being transmitted through paternal gametes, our significant observations were restricted to maternally affected pedigrees. There are also observations in monogenic disorders that suggest maternal transmission effects. For example, congenital $\mathrm{MD}$ appears to be exclusively a consequence of maternal transmission. ${ }^{1}$ In fragile $\mathrm{X}$ syndrome, expansion of the premutation occurs only through the maternal line. ${ }^{12}$
There could be alternative explanations for these observations, other than premutation. Other epigenetic phenomena, such as imprinting, or differential $\mathrm{X}$ chromosome inactivation may be operative. ${ }^{13}$ Maternal in utero effects which are age related could be involved. It seems unlikely that secular trends in disease could account for our observations, given the epidemiological evidence that RA is becoming less common and less severe. ${ }^{14}$

Our observations on early disease onset, increased disease severity, and familiarity might have been accounted for by an enrichment of HLA-DR4 in the pedigrees. ${ }^{15}$ This mechanism was not a predominant factor in the multicase pedigrees. The prevalence of HLA-DR4 was no greater than we would expect for a population identified by means of hospital records, and the transmitted HLA-DR haplotype contained DR4 in only four of the seven cases.

These preliminary data need to be confirmed in larger, fully documented samples. Lessons learned in the context of monogenic disorders may be of relevance to subgroups of more complex diseases.

Dr C M Deighton was supported by a Medical Research Council Travelling Fellowship. Dr G Thomson is supported by NIH Grant HD12731.

1 Harper P S, Harley H G, Reardon W, Shaw D J Anticipation in myotonic dystrophy: new light on an old problem. Am f Hum Genet 1992; 51: 10-16.

2 Ross C A, McInnis M G, Margolis R L, Li S H. Genes with triplet repeats: candidate mediators of neuropsychiatric disorders. Trends Neurosci 1993; 16: 254-60.

3 Zheng C-J, Byers B, Moolgavkar S H. Allelic instability in mitosis: a unified model for dominant disorders. Proc Natl Acad Sci USA 1993; 90: 10178-82.

4 McInnis M G, McMahon F J, Chase G A, Simpson S G, Ross C A, DePaulo J R. Anticipation in bipolar affective disorder. Am F Hum Genet 1993; 53: 385-90.

5 Zheng C-J, Peng Y-N, Thomson G. Allelic instability in mitosis can explain "genome imprinting" and other genetic phenomena in psoriasis. Am $\mathcal{F}$ Med Genet 1994. In press.

6 Arnett F C, Edworthy S M, Bloch D A, et al. The American Rheumatism Association 1987 revised criteria for the classification of rheumatoid arthritis. Arthritis Rheum 1988; 31: 315-24.

7 Cobb S, Thompson D J, Rosenbaum J, Warren J E, Merchant W R. On the measurement of prevalence of arthritis and rheumatism from interview data. $f$ Chron Dis 1956; 3: 134-44.

8 Walker D J, Burn J, Griffiths I D, Roberts D F, Stephenson A M. Linkage studies of HLA and rheumatoid arthritis in multicase families. Arthritis Rheum 1987; 30: 31-5.

9 Walker D J, Griffiths I D. HLA associations are with severe rheumatoid arthritis. Dis Markers 1986; 4: 121-32.

10 Kirwan J R, Reeback J S. Stanford Health Assessment Questionnaire modified to assess disability in British patients with rheumatoid arthritis. Br f Rheumatol 1986; 25: 206-9.

11 Dewar P J. HLA antigens. Clin Rheum Dis 1983; 9: 103-5.

12 McConkie-Rosell A, Lachiewicz A M, Spiridigliozzi G A, et al. Evidence that methylation of the FMR-1 locus is responsible for variable phenotypic expression of the fragile X syndrome. Am f Hum Genet 1993; 53: 800-9.

13 Gregersen $P \quad K$. Discordance for autoimmunity in monozygotic twins. Are "identical" twins really identical? Arthritis Rheum 1993; 36: 1185-92.

14 Silman A J. Trends in the incidence and severity of rheumatoid arthritis. F Rheumatol 1992; 19(suppl 32): $71-3$.

15 Goronzy J J, Weyand C M. Interplay of T lymphocytes and HLA-DR molecules in rheumatoid arthritis. Curr Opin Rheumatol 1993; 5: 169-77. 